\title{
La práctica reflexiva y la infografía como recurso didáctico
}

\section{Paola Ruiz-Bernardo ${ }^{a}$, Sonia Vecino Ramos ${ }^{b}$ y José Luis Rambla Nebot ${ }^{c}$}

${ }^{\mathrm{a}}$ Universidad Jaume I (Castellón, ruizma@uji.es), ${ }^{\mathrm{b}}$ Universidad Jaume I (Castellón, al400470@uji.es) y ${ }^{\mathrm{c}}$ Universidad Jaume I (Castellón, jorambla@uji.es)

\begin{abstract}
Reflective practice is a quality aspect of higher education. Therefore, this innovative proposal seeks to promote spaces and resources to make reflection effective in University classrooms. This communication intends to relate an experience located at Jaume I University in Castellón, in the subject of school organization, in the first year of the Bachelor's Degree of Teaching (Nursery and Primary).

The methodology has been centered on infographics (vignettes) as a tool to initiate reflection and debate within the class group.

The results collected from the students have been highly positive both at the level of reflection and learning outcomes and also as personal satisfaction with the work done. This is the reason why this experience has been suggested to be continued in successive years, and its transference to other subjects has been considered.
\end{abstract}

Keywords: Educational innovation, Self-learning, Reflective practices, Active methodologies, Higher education, Transversal competences.

\section{Resumen}

La práctica reflexiva es un aspecto que otorga calidad a la enseñanza de nivel superior. Por ello, mediante esta propuesta innovadora se busca promover los espacios y los recursos para hacer efectiva la reflexión en las aulas universitarias. Esta comunicación pretende relatar una experiencia situada en la Universitat Jaume I de Castellón, en el Grado de Maestros/as (de Infantil y Primaria) en la asignatura de organización escolar, de primer curso.

La metodología se ha centrado en el uso de la infografía (viñetas) como herramienta para iniciar la reflexión, seguida del debate dentro del grupo clase.

Los resultados recogidos de las voces de los alumnos han sido muy positivos tanto a nivel de reflexión y resultados del aprendizaje, así como de satisfacción personal con el trabajo realizado, por lo cual se sugiere la continuidad de la experiencia en años sucesivos y su posible transferencia a otras asignaturas.

Palabras clave: Innovación educativa, Autoaprendizaje, Prácticas reflexivas, Metodologías activas, Educación superior, Competencias transversales. 


\section{Introducción}

La innovación educativa se entiende como la capacidad de realizar cambios en el aprendizaje/formación que produzcan mejoras en los resultados de aprendizaje. Sin embargo, para que se considere innovación educativa el proceso debe responder a unas necesidades, debe ser eficaz y eficiente, además de sostenible en el tiempo y con resultados transferibles más allá del contexto particular donde surgieron (GarcíaPeñalvo, 2015).

La actual propuesta surge como una mejora en la asignatura de Organización Escolar del grado de Maestro/a de Infantil y Primaria de la Universidad Jaume I de Castellón. Después de varios años de experiencia docente se ha observado que existe, en la universidad en general, la preocupación por la ausencia y falta de integración de conocimientos, tanto entre los contenidos de la asignatura como de toda la carrera, a lo que se suma la desvinculación de lo que se estudia con los problemas sociales que nos rodean, lo cual produce un aprendizaje fragmentado y poco significativo.

Además, también se percibe que el perfil del estudiantado universitario ha cambiado como consecuencia del impacto y desarrollo de las nuevas tecnologías, así como de la importancia cada vez mayor de la cultura visual.

Como respuesta a estas y otras inquietudes docentes, esta experiencia muestra las ventajas de incorporar la infografía en el aula como herramienta que permite aprovechar las potencialidades de la imagen, que nos sugiere un pensamiento sin utilizar las palabras.

La infografía es un recurso metodológico útil dada su versatilidad y posibilidad para el aprendizaje significativo. Siguiendo a Morera (2017) se entiende que "la infografía es un lenguaje visual, formado por texto e imagen en el que ambos lenguajes trabajan al unísono para ofrecer información por el canal visual" (p. 328).

La infografía didáctica como recurso visual y lingüístico ya ha sido aplicada en numerosos contextos educativos, puesto que es considerada como una herramienta transversal de cognición que permite la gestión del conocimiento, la capacidad de síntesis y la esquematización de contenidos (Albar, 2016). El pensamiento crítico relativo a lo social no sólo contextualiza los contenidos de la asignatura sino que potencia el desarrollo de competencias sociales y reflexivas.

\section{La asignatura y su metodología}

La asignatura Organización Educativa pretende que el alumnado adquiera competencias relacionadas con la organización y gestión de los centros educativos, pues su contexto de trabajo no solamente será en el aula, sino el centro educativo y, en un ámbito más amplio, la comunidad educativa. Además, la tarea del maestro o maestra con niños y niñas está muy determinada, tanto por la legislación vigente como por los documentos organizativos que regulan la vida del centro. Un determinado tipo de organización escolar refleja las ideas vigentes sobre la sociedad, sobre la persona que educa, sobre la naturaleza de la enseñanza-aprendizaje y sobre la función de la escuela en la sociedad.

Partiendo de la idea de que la escuela es una realidad compleja, es necesario que el estudiantado se familiarice con todos estos conceptos y sea capaz de reflexionar críticamente ante toda la complejidad que rodea la profesión. No solo es importante conocer el centro escolar, su funcionamiento y sus miembros, sino que el alumnado ha de aprender a crear un buen clima organizativo que ayude a la mejora de la calidad de los procesos de enseñanza-aprendizaje. El futuro maestro o maestra ha de conocer los modelos organizativos que permiten adaptar las diversas propuestas metodológicas. 
La metodología de esta asignatura se rige por las competencias y contenidos establecidos, previamente en los documentos de verificación del título del grado. Tabla 1.

Tabla 1: Competencias a desarrollar en la asignatura.

\section{Competencias genéricas y específicas}

FB09 - Analizar la práctica docente y las condiciones institucionales que la enmarcan.

FB10 - Conocer el sistema educativo actual de nuestro país y los condicionantes políticos y legislativos de la actividad docente.

FB18 - Participar en la definición del proyecto educativo y en la actividad general del centro atendiendo a criterios de gestión y calidad.

FB21 - Conocer y saber ejercer las funciones de tutor en relación al individuo y a las familias.

FB23 - Analizar e incorporar de forma crítica las cuestiones más relevantes de la sociedad actual que afectan a la educación, familiar y escolar.

Fuente: Guía docente de la asignatura.

En cuanto a la metodología, se trata de fomentar el debate y la reflexión crítica por parte del alumnado, mediante ejemplos y casos que den lugar a diferentes puntos de vista. Para ello se combinan diversas técnicas: desde el trabajo individual, al trabajo por parejas, o las técnicas grupales cooperativas como el puzzle de Aronson, el juego de DeVries, los seis sombreros para pensar, etc. A la vez, se plantea otro tipo de metodología, como por ejemplo, el aprendizaje basado en problemas (ABP) o el estudio de casos.

\section{Objetivos}

Los objetivos generales y específicos sobre los que se centró la experiencia fueron:

1. Aplicar la infografía (viñeta) como herramienta para facilitar el diálogo y la reflexión entre los equipos de trabajo durante diferentes momentos en el transcurso de la asignatura.

1.1. Extraer los conocimientos previos a la asignatura.

1.2. Lograr la integración de los contenidos de la asignatura.

1.3. Ser autónomos en la gestión de su aprendizaje.

1.4. Debatir los contenidos y reflexionar conjuntamente.

2. Evaluar la efectividad de esta herramienta, valorando si resulta útil para obtener un aprendizaje más integrado, global y reflexivo.

\section{Desarrollo de la innovación}

Estas asignaturas están ambas ubicadas en el primer curso de las carreras mencionadas, y han participado en esta experiencia metodológica cuatro grupos de Prácticas (200 alumnos aproximadamente). Los datos que se han recogido corresponden a dos cursos académicos consecutivos, con lo cual el grupo total destinatario de esta metodología y posterior evaluación han sido unos 400 alumnos, durante los cursos académicos 2018-2019 y 2019-2020.

Durante diferentes momentos de la docencia de la asignatura se ha utilizado esta metodología. En el inicio, para hacer un diagnóstico de lo que los alumnos conocían de la realidad social y educativa, y de nuevo al final de la asignatura, para iniciar el escrito de un ensayo inspirado en la imagen pero con la idea de integrar todos los contenidos revisados en la asignatura. 
Esta actividad, en ambos momentos, se hace en grupos pequeños (entre 4 y 5 personas) para favorecer un aprendizaje dialógico (Moliner, Sanahuja y Benet, 2017) mediante el contraste de ideas y conceptos que la imagen hace surgir de sus conocimientos previos.

Esta metodología está inspirada en el capítulo de "dejar que los libros hablen" del libro de Don Finkel (2008), ya que su propuesta es que al utilizar un recurso, que en su caso puede ser un libro clásico o una parábola, éste ayude a aflorar una reflexión que no responda a la imagen descriptiva que se expresa sino que el alumno sea capaz de sacar el significado oculto y nunca único de lo que expresa. En el caso que relatamos esto se realiza partiendo de factores condicionantes en el entorno de los estudiantes como son: los pocos hábitos de lectura, la escasez del tiempo para leer en clase y, por último, la imagen y la vista como el principal iniciador de los procesos cognitivos para la reflexión.

Para Finkel (2008) la selección del recurso es esencial, ya que los hechos deben ser reales y posibles pero al mismo tiempo no deben dar un resultado definitivo (son opacos). Además añade que el profesor debe crear las circunstancias para una experiencia de aprendizaje basada en el análisis y la reflexión sobre el recurso propuesto.

Por esta razón y siguiendo los puntos señalados por Finkel, para esta asignatura se han utilizado como recurso visual (infografía) imágenes que se obtienen de viñetas de diferentes autores y que son de acceso gratuito. En cuadro siguiente (Cuadro 1) se muestra un ejemplo:

Cuadro 1: Ejemplo de recurso visual para favorecer la reflexión e integración de contenidos.

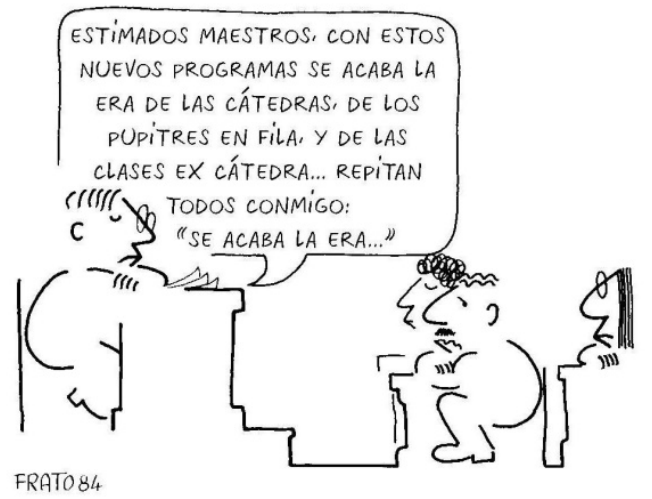

Por tanto, la metodología consiste en distribuir la clase en grupos, entre los cuales se reparten al azar las diferentes viñetas, que son todas diferentes, y se les propone que escriban un ensayo a partir de un modelo que está colgado en el aula virtual. También se les propone una secuencia de pasos para favorecer el desarrollo del pensamiento reflexivo personal, es decir, hacer el primer trabajo de modo individual en una ficha que proporciona la docente $y$, en un segundo momento, leer al grupo todas la ideas individuales para, al final, elaborar juntos un solo ensayo que contenga el conjunto de voces de modo armónico.

Una vez finalizado, ponemos en marcha otra de las propuestas de Don Finkel (2008): “dejar que los alumnos hablen". En esta situación son los alumnos los que mediante la escritura expresan en sus ensayos sus aprendizajes, pero estos serán leídos y evaluados por los otros grupos de la clase (coevaluación). En este caso cada alumno hace un contraste de sus aprendizajes mediante la lectura de otros trabajos y nuevamente se repite la secuencia: una reflexión individual que luego comparte con el grupo. Lo novedoso es que se genera una respuesta del grupo lector o evaluador al grupo que escribe mediante una carta donde se expresan: su satisfacción con el escrito, las felicitaciones por los puntos fuertes, y sus sugerencias de mejoras (incorporando contenidos que no se han tenido en cuenta). Por último, el grupo 
evaluador propone una calificación que corrobora el docente de la clase. Hay que decir que para ello existe una rúbrica (Anexo 1) que sirve de guía para evitar apreciaciones subjetivas.

\section{Resultados}

Al finalizar la docencia de la asignatura se consultó a los alumnos (mediante una encuesta anónima, diseñada con la herramienta de Formularios Google) sobre la satisfacción con las técnicas y si tenían experiencia previa en estos tipos de trabajo.

1- La muestra de alumnos se puede observar en la figura 1, donde además se especifica la asignatura que han cursado (MP1846: Organización Escolar de Primaria, MI1861: Organización Escolar de Infantil).

\section{Grado-Asignatura}

207 respuestas

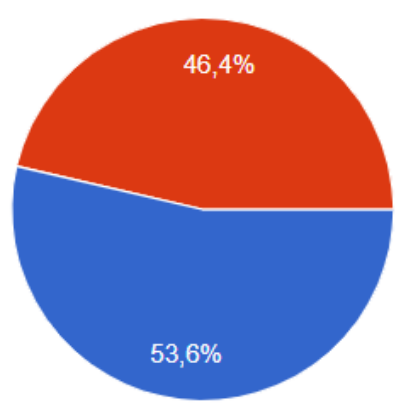

Figura 1: Información sobre la muestra de alumnos de las asignaturas. Fuente: elaboración propia.

2- Resultados de satisfacción y percepción de mejora de sus competencias y adquisición de conocimientos (Figura 2).

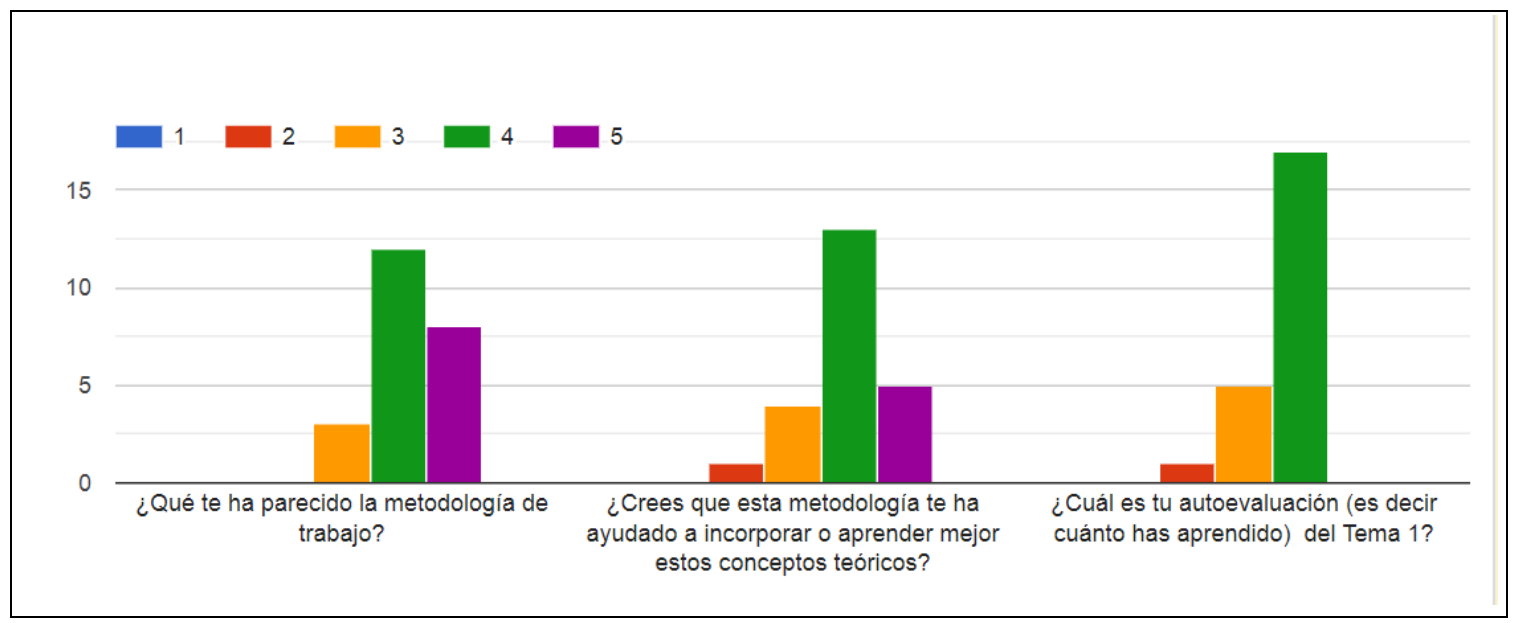

Figura 2: Satisfacción de los alumnos. 
Así también, al evaluar el resultado del aprendizaje, que se observa en la integración de los contenidos volcados en el ensayo que han escrito, resulta muy satisfactorio por el nivel reflexivo que alcanzan. En el pasado curso, la nota media de estos ensayos osciló entre el 8,50 y el 10.

3- En este último apartado se presentan los resultados ante la pregunta de si había participado antes en alguna actividad semejante (Figura 3). El objetivo de esta pregunta es evidenciar que se trata de una innovación, en tanto que esto es una novedad para ellos que les permite aprender, mediante esta técnica, una reflexión que vincule los contenidos y el pensamiento crítico sobre la sociedad.

¿Has participado anteriormente en alguna actividad que sea semejante a esta?

207 respuestas

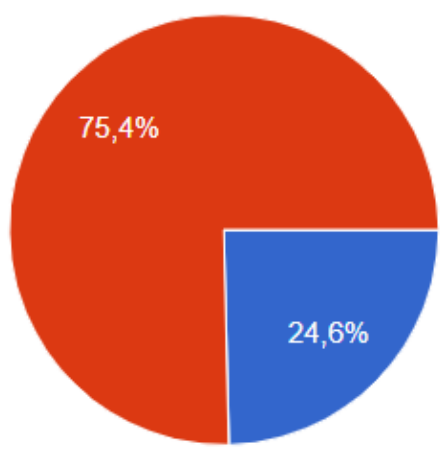

Figura 3: Experiencia previa de los alumnos en relación a las técnicas utilizada. Fuente: elaboración propia.

\section{Conclusiones}

La práctica reflexiva en el aula es posible si se desarrolla lo que Finkel (2008) denomina "circunstancias" que la posibiliten. Vemos que la secuencia de pasos a seguir, el tiempo del aula aprovechado para que los alumnos hablen, y el contraste de la mirada del resto el grupo con el individuo y entre los propios grupos, se constituyen como esas circunstancias que complementan muy bien la técnica de la infografía. La razón es que esto provoca que el alumno explore en su interior para extraer sus conocimientos previos o para plasmar los contenidos aprendidos.

En relación a los objetivos planteados, la infografía se propone como una herramienta que facilita un aprendizaje más reflexivo, significativo y eficaz, lo cual está relacionado con las dinámicas grupales de trabajo, la resolución de conflictos, las habilidades comunicativas, etc. Por lo tanto, mediante el uso de esta práctica los alumnos no sólo aprenden los contenidos de la asignatura, sino que desarrollan otras competencias transversales muy importantes para su vida laboral futura.

Por ello es apropiado remarcar que, dado que se trata de una actividad simple pero muy efectiva, es transferible a otros ámbitos tales como la economía o cualquier otra de las ciencias sociales. 


\section{Referencias}

ALBAR, P. (2016). Infografía didáctica, un recurso visual y lingüístico implementado como herramienta visual transversal de cognición a través de la educación artística. En González y Castro, Democracia y Educación en el siglo XXI. La obra de John Dewey 100 años después: libro de actas del XVI Congreso Nacional y VII Congreso Iberoamericano de Pedagogía en la Facultad de Educación de la Universidad Complutense de Madrid, España.

CARRIÓN, E. (2014). Los medios audiovisuales y las TIC como herramientas para la docencia en educación secundaria. Análisis aplicado de una práctica docente. Ensayos: revista de la Facultad de Educación de Albacete, 94(2), 37-62.

FINKEL, D.(2008). Dar clases con la boca cerrada. Traducción de Barberá, O. Universidad de Valencia. España.

GARCÍA-PEÑALVO, F. (2015). Mapa de tendencias en Innovación Educativa. Education in the Knowledge Society, 16(4),6-23.[fecha de Consulta 11 de Marzo de 2020]. ISSN: Disponible en: https://www.redalyc.org/articulo.oa?id=5355/535554760001.

LEÓN, G. (2010). La formación interdisciplinaria de los profesores. Una necesidad del proceso de enseñanza y aprendizaje de las ciencias. Ensayos pedagógicos, 5(1), 119-130.

MORERA, FJ. (2017). Aproximación a la infografía como comunicación efectiva. Tesis doctoral, Universitat Autònoma de Barcelona, España. Morin, E. (1998). Sobre la interdisciplinariedad. Boletín 2 del Centre Internacional de Recherches et Etudes Transdisciplinaires (CIRET).

MOLINER GARCÍA, O., SANAHUJA RIBÉS, A., \& BENET-GIL, A. (2017). Prácticas inclusivas en el aula desde la investigación-acción. http://repositori.uji.es/xmlui/handle/10234/168902.

\section{Anexo 1}

Rúbrica de evaluación de los trabajos escritos y reflexivos de los grupos de clase.

\begin{tabular}{|l|l|l|l|l|l|}
\hline & Excelente (9-10) & Bien (7-8) & Regular (5-6) & Mal (2-4) & Pésimo (0-1) \\
\hline $\begin{array}{l}\text { Claridad y } \\
\text { organización de } \\
\text { las ideas }\end{array}$ & $\begin{array}{l}\text { La estructura es muy } \\
\text { clara y se entiende } \\
\text { perfectamente }\end{array}$ & $\begin{array}{l}\text { La organización está } \\
\text { clara y casi todas las } \\
\text { ideas conectadas }\end{array}$ & $\begin{array}{l}\text { Hay claridad en la } \\
\text { redacción pero las } \\
\text { ideas están } \\
\text { desconectadas }\end{array}$ & $\begin{array}{l}\text { Se salta de una idea a } \\
\text { otra sin una conexión } \\
\text { clara entre ellas }\end{array}$ & $\begin{array}{l}\text { No se entiende } \\
\text { nada }\end{array}$ \\
\hline Nivel de reflexión & $\begin{array}{l}\text { Ha profundizado en } \\
\text { todos los aspectos clave } \\
\text { del tema }\end{array}$ & $\begin{array}{l}\text { Detecta y enumera } \\
\text { los aspectos clave y } \\
\text { profundiza en algunos }\end{array}$ & $\begin{array}{l}\text { Detecta y enumera los } \\
\text { aspectos clave pero no } \\
\text { profundiza en ellos }\end{array}$ & $\begin{array}{l}\text { Hace un resumen de las } \\
\text { lecturas o las } \\
\text { aportaciones }\end{array}$ & No se reflexiona \\
\hline $\begin{array}{l}\text { Conclusiones } \\
\text { sobre el } \\
\text { aprendizaje }\end{array}$ & $\begin{array}{l}\text { Hace transferencias } \\
\text { entre las lecturas y } \\
\text { ensayos su experiencia y } \\
\text { la vida real }\end{array}$ & $\begin{array}{l}\text { Hace algunas } \\
\text { transferencias con su } \\
\text { experiencia y la vida } \\
\text { real }\end{array}$ & $\begin{array}{l}\text { pero no son correctas } \\
\text { peransferencias }\end{array}$ & $\begin{array}{l}\text { Los aprendizajes } \\
\text { descritos son } \\
\text { transcripciones literales } \\
\text { de los ensayos } \\
\text { presentados }\end{array}$ & $\begin{array}{l}\text { No hay ninguna } \\
\text { evidencia de }\end{array}$ \\
\hline
\end{tabular}

